\title{
Effect of Silica Morphology on the Structure of Hard- Templated, Non-Precious Metal Catalysts for Oxygen Reduction
}

Jacob Anibal ${ }^{1}$, Henry G. Romero ${ }^{2}$, Nathaniel D. Leonard ${ }^{1}$, Cenk Gumeci ${ }^{1}$, Barr Halevi ${ }^{2}$, Scott Calabrese Barton ${ }^{1 *}$

${ }^{1}$ Department of Chemical Engineering and Materials Science, Michigan State University, East Lansing, MI 48824(USA)

${ }^{2}$ Pajarito Powder, Albuquerque, NM 87102 (USA)

\section{Abstract}

With extensive development over the past ten years, non-precious metal (NPM) catalysts have become promising alternatives to platinum catalysts in fuel cell cathodes, but have been challenged by severe transport limitations. Hard template synthesis, also called the sacrificial support method, promises to improve transport properties introducing a sacrificial template to form mesopores. To further understand hard template transport properties, the relationship between template morphology, resulting catalyst pore structure, and overall fuel cell performance was investigated. Porous carbon materials and catalysts were synthesized using four different silica templates. Nitrogen physisorption and scanning electron microscopy were used to characterize pore diameter and morphology. The catalysts displayed pores approximately half the diameter of the template particles, and a geometric model is proposed to describe pore collapse. Catalyst electrochemical performance was characterized in membrane electrode assemblies using

\footnotetext{
* Corresponding Author. E-mail: scb@msu.edu (Scott Calabrese Barton), tel: +1-517-355-0222
} 
the hard templated materials in gas-diffusion electrodes for oxygen reduction. An optimum pore diameter was observed at approximately $9 \mathrm{~nm}$.

Keywords: Fuel cells; Oxygen reduction reaction; Non-precious catalysts; Pore size distribution; Membrane-Electrode Assembly.

\section{Introduction}

Increasing petroleum prices within the last decade have drastically increased interest in fuel cells as an alternative technology. Despite the high energy density and efficiency imparted by platinum electrocatalysts, the cost of the precious metal continues to limit the feasibility of fuel cells in vehicle powertrains. Metal-Nitrogen-Carbon (MNC) catalysts produced using high temperature pyrolysis offer a promising, inexpensive alternative to platinum catalysts. Despite their lower costs, MNC catalysts have not found widespread use due to their low activity relative to platinum [1,2]. Many researchers have contributed to improved performance, but MNC catalysts still have performance well below platinum [3].

Recently, a key focus of MNC development has been enhancement of transport within the carbon support structure [4,5]. Producing a catalyst with optimum transport properties requires a synthesis technique with control over a catalyst's physical properties. Hard templating is one such technique. Developed in the late 1980's [6], hard template synthesis controls the pore size, shape and structure via a template particle.

Hard templating consists of a three to four step process. The process begins with the deposition of an organic precursor onto template particles. The template is often either silica particles or polymer materials. After deposition, the precursor is decomposed by pyrolysis to 
form a carbonaceous material over the template surface. The template is then removed, either by chemical reagents or high temperatures, leaving a highly porous, carbon product. An additional carbonization step often follows template removal to fully solidify the porous carbon material. More extensive reviews of hard templating are given by Lee et al [7] and Xia et al [8].

Pylypenko and coworkers extended the hard template process to catalyst synthesis. They used a sacrificial support method to produce a metal-nitrogen-carbon catalyst from metal macrocycles [9]. The hard template catalysts showed excellent oxygen reduction activity and a high degree of mesoporosity. Subsequent work by researchers in the Atanassov group improved catalyst activity by optimizing process parameters and varying the carbon-nitrogen precursor [10-14]. Despite the promise of hard template catalysts, little is known about their physical structure or its effect on catalyst performance. This work investigates the relationship between hard template catalyst pore structure and silica precursor morphology. Four hard template catalysts were synthesized using silica particles with modal diameters between 14 and $40 \mathrm{~nm}$. Catalyst pore structure was analyzed using nitrogen physisorption and scanning electron microscopy and compared with the precursor structure. Membrane electrode assembly (MEA) tests followed physical characterization to relate catalyst performance and structure.

\section{Experimental}

\subsection{Synthesis}

Porous carbon materials and catalysts were synthesized by Pajarito Powder, Inc. using a proprietary technique based on the methodology of Serov et al [12]. Briefly, porous carbon materials were prepared by mixing a carbon-nitrogen precursor with fumed silica particles, 
followed by pyrolysis in inert atmosphere $\left(900{ }^{\circ} \mathrm{C}, 1\right.$ hour $)$. The silica was then etched with hydrofluoric acid and a second pyrolysis occurred in inert atmosphere. Nicarbazin was used as the carbon-nitrogen precursor in all syntheses [14]. Catalysts were synthesized in the same manner as the porous carbon materials, but had iron nitrate added to achieve $20 \mathrm{wt} \%$ Fe prior to the first pyrolysis. Following etching, the resulting iron content was $0.5 \mathrm{wt} \%$. Porous carbon materials and catalysts were synthesized using four different fumed silicas: Evonik Aerosil@ OX-50, Cabot Cab-O-Sil@ L90, Cab-O-Sil® LM150, and Cab-O-Sil® M5P, respectively. Figure 1 summarizes the silica particle properties as reported by the manufacturers. The silica particles show moderate specific surface areas which generally agree with geometric predictions for hard body spheres. The L90 silica does deviate from the theoretical trend, possibly due to greater fusion between the silica particles. Boldridge gives an in-depth geometric characterization of the L90 silica [15].

\begin{tabular}{ccc}
\hline Silica & $\begin{array}{c}\text { Particle } \\
\text { Diameter } \\
(\mathbf{n m})\end{array}$ & $\begin{array}{c}\text { Surface } \\
\text { Area } \\
\left(\mathbf{m}^{2} \mathbf{~ g}^{-1}\right)\end{array}$ \\
\hline Aerosil@ OX-50 [24] & 40 & $50 \pm 15$ \\
Cab-O-Sil® L-90 [24] & 20 & $90 \pm 10$ \\
Cab-O-Sil® LM-150 [25] & 18 & $160 \pm 15$ \\
Cab-O-Sil® M-5P [24] & 14 & $200 \pm 15$ \\
\hline
\end{tabular}

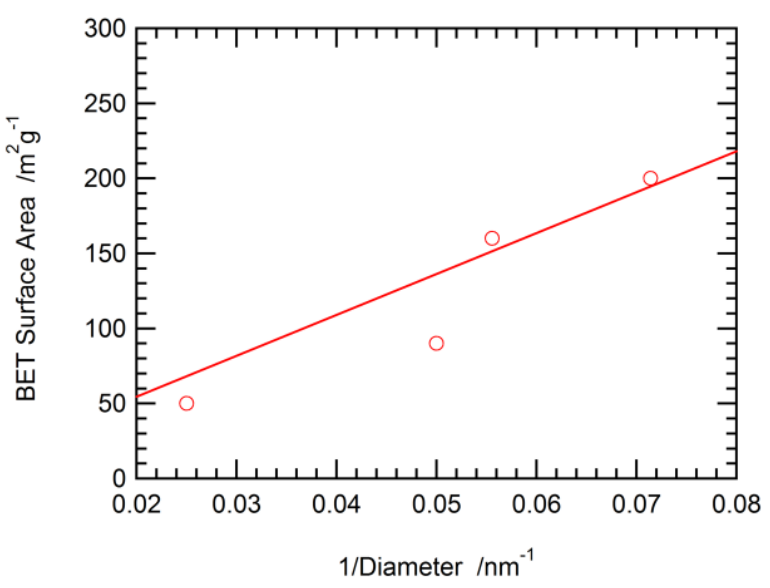

Figure 1. Summary of the silica particle properties. The plot shows the relationship between silica surface area and particle diameter with a comparison to the theoretical geometric relationship for spheres. 


\subsection{Physical Characterization}

Nitrogen adsorption isotherms for the hard template materials were obtained using a Micromeritics ASAP 2020 Nitrogen adsorption analyzer. The surface area of the porous carbons and catalysts was measured using the Brunauer-Emmett-Teller (BET) methodology [16]. Pore size distributions were obtained from the isotherms using the Barrett-Joyner-Halenda (BJH) [17] and Nonlocal Density Functional Theory (DFT) [18,19] approaches. BJH calculations were performed using the desorption branch of the isotherm, while DFT used the adsorption branch.

Scanning electron microscopy (SEM) was performed using a JEOL 7500F ultra-high resolution scanning electron microscope equipped with Scandium image processing software. Each specimen was mounted on conductive carbon tape atop a $15 \mathrm{~mm}$ aluminum stub. Materials were imaged at $5 \mathrm{kV}$ with a $20 \mu \mathrm{A}$ emission current and a typical working distance of $5 \mathrm{~mm}$. All hard-templated materials (porous carbon and catalyst) were conductive and did not require coating.

\subsection{MEA Fabrication and Testing}

Catalyst layers were constructed by spraying catalyst ink onto a $5 \mathrm{~cm}^{2}$ Sigracet $25 \mathrm{BC}$ GDL using a Sono-tek Exacta-Coat automated spray system. The ink contained $500 \mathrm{mg}$ catalyst per $20 \mathrm{~mL}$ solvent with a 9:11 mass ratio of Nafion to catalyst. After drying, the catalyst coated GDL was assembled with a 211 Nafion membrane and $0.2 \mathrm{mg}_{\mathrm{Pt}} / \mathrm{cm}^{2}$ anode to form the Membrane Electrode Assembly (MEA). Following assembly, the MEAs were hot pressed at $131^{\circ} \mathrm{C}$ and 90 psi for six minutes. 
Following fabrication, MEAs were sandwiched between two, single serpentine pattern flow plates and tightened with $4.5 \mathrm{Nm}$ torque to form fuel cells for performance testing. Tests were performed using Fuel Cell Technologies (Albuquerque NM) test stands at $80^{\circ} \mathrm{C}$, with 30 psi back pressure, and a feed rate of $200 \mathrm{sccm}$. Prior to testing, each cell was broken in by holding the potential at 0.3 and $0.6 \mathrm{~V}$ for 15 minutes each. MEA performance was measured potentiostatically, with potentials ranging from open circuit to $0.25 \mathrm{~V}$, and with 60 seconds stabilization time before recording the cell current.

\section{Results and Discussion}

Nitrogen physisorption characterization of the porous carbons and catalysts indicated highly porous materials, with total adsorbed Nitrogen above $400 \mathrm{~cm}^{3} / \mathrm{g} \mathrm{STP}$ near saturation pressure, as shown in Figure 2. All of the hard template materials displayed type IV isotherms with significant hysteresis loops [20]. 


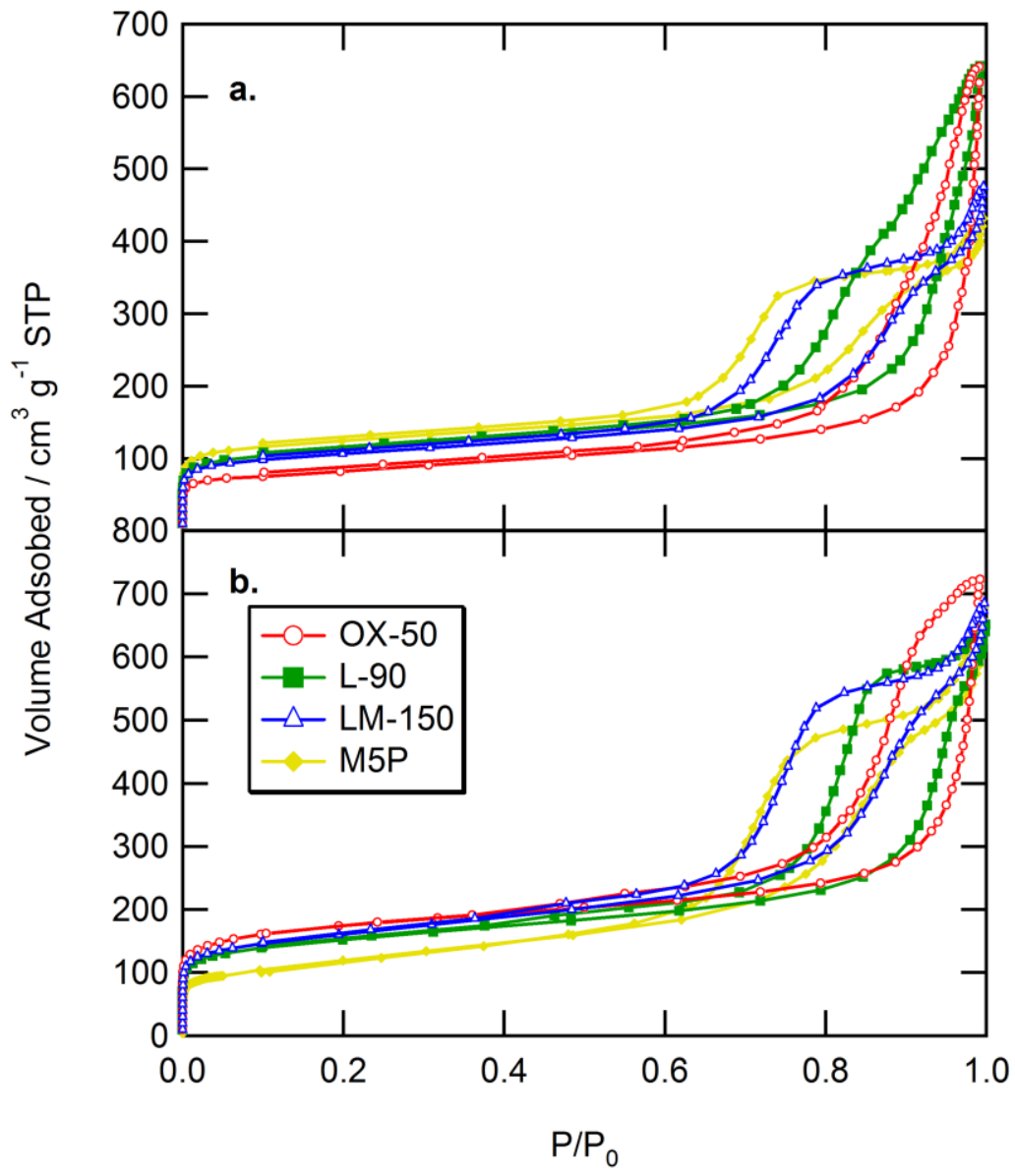

Figure 2. Nitrogen adsorption-desorption isotherms for (a) porous carbon materials and (b) catalysts.

BJH and DFT pore size distributions were obtained from the nitrogen adsorption isotherms, as shown in Figure 3. The BJH pore size distributions displayed broad peaks in the mesoporous range. The peak locations varied among the different precursors, but were nearly identical for each porous carbon-catalyst pair, indicating a very weak effect of iron nitrate on the hard template's structure. The iron precursor's only noticeable effect was a slight decrease in catalyst mesoporosity, most noticeable for the L-90 catalyst. The pore size distribution widths 
also offered insight into the pore formation mechanism. The distribution widths correlated strongly with pore size. Fumed silica particle distributions follow a similar correlation, widening with increasing particle size [21]. This similarity reflects the expected direct impact of silica particle size on catalyst pore size.

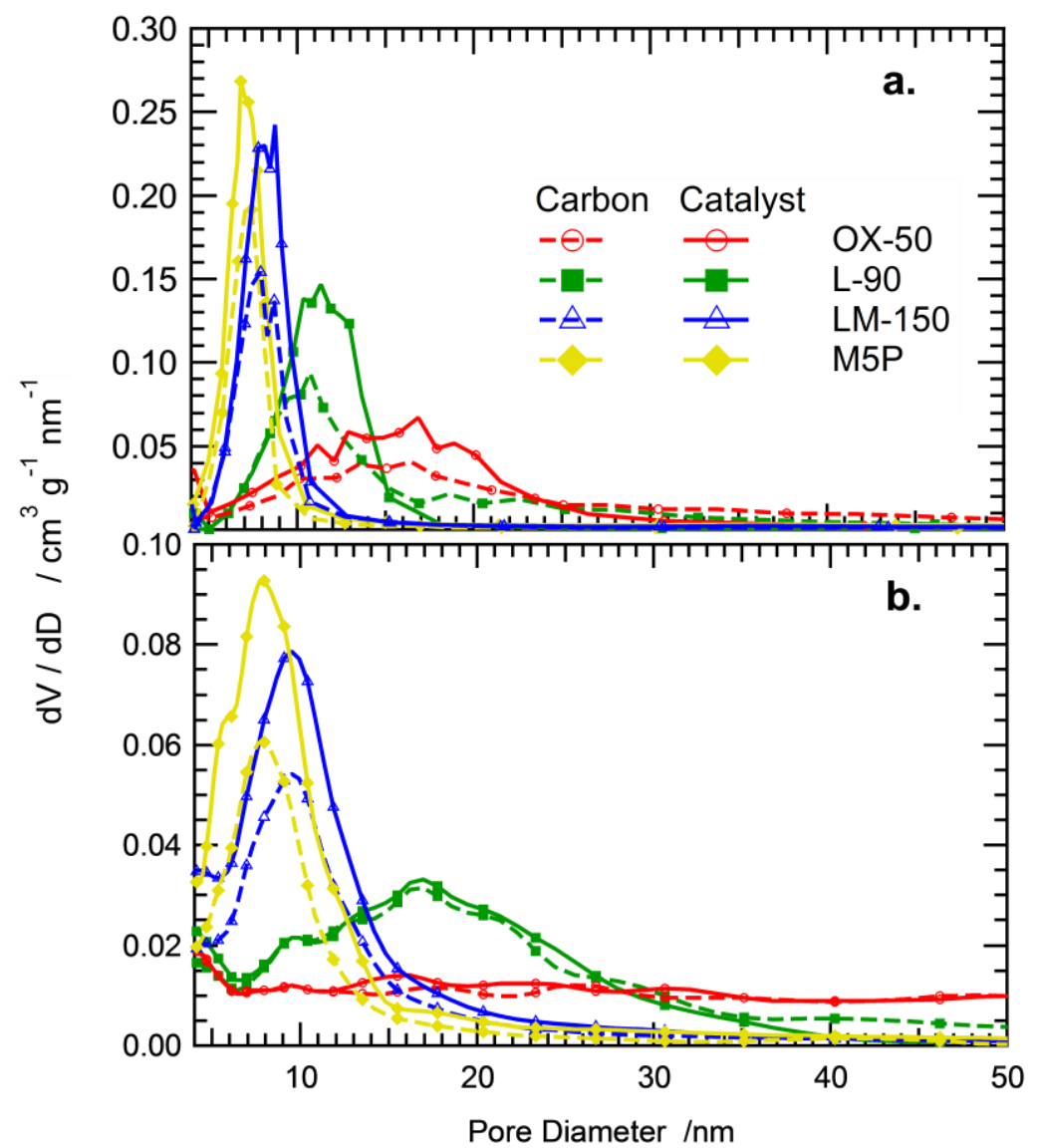

Figure 3. Pore size distributions for the hard template materials obtained using the (a) BJH and (b) DFT methodologies.

The DFT pore size distributions displayed similar general characteristics to the BJH distributions, exhibiting unimodal peaks in the mesoporous region. The DFT distribution also 
showed large, sharp peaks around two nanometers, indicating significant microporosity. These microporous peaks were excluded from Fig. 3 to allow viewing of the mesopore peaks. Appendix B (Supporting Information) presents the micropore distributions. The main DFT and BJH peaks were centered at similar pore diameters, with the DFT displaying slightly larger values. The L90 catalyst proved the exception, displaying a repeatable major DFT pore size more than five nm larger than the BJH distribution. The DFT distributions also displayed considerably wider peaks than the BJH distributions. Part of the BJH-DFT discrepancy can be attributed to the difference between the adsorption and desorption isotherm branches (Fig. 2). Traditionally, hardtemplate carbon materials have been known to form significant quantities of dead end pores [22], presenting the possibility of an "ink bottle" pore effect [23] in the desorption-based BJH distributions. This effect would result in narrower distributions, as the volume of larger pores would be attributed to smaller, bottleneck pores. Ink bottle-type pores would also cause a shift in the pore size distribution toward the size of the bottleneck pore.

The hard template pore diameter correlated strongly with silica template particle diameter, as shown in Figure 4. The BJH pore diameter values showed a strong, positive correlation with the silica particle diameter. The DFT values display a weaker positive correlation. Both the BJH and DFT distributions reveal pore diameters significantly smaller than the silica particles, on the order of one half. The decrease in pore diameter could result from pore collapse during either silica etching or the second heat treatment. 


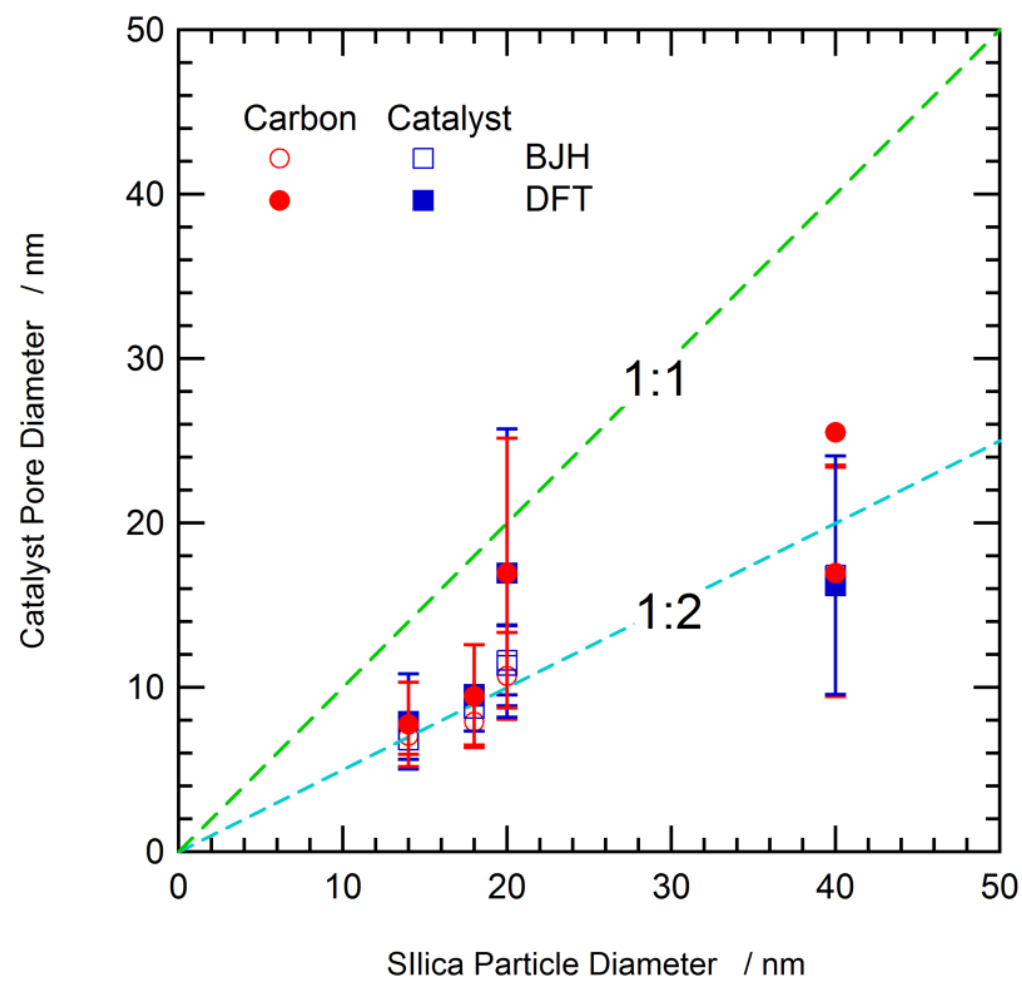

Figure 4. Comparison of the hard template pore size and precursor particle size. The error bars represent the width at half the maximum peak height.

In addition to high porosity, the hard template materials also displayed good surface areas, over $300 \mathrm{~m}^{2} \mathrm{~g}^{-1}$, as shown in Figure 5. The surface areas were on par with those reported for previous hard template catalysts [9]. The porous carbon's surface area correlated with the silica precursor area, but was generally much higher. This increase in surface area could result from the creation of micropores and defects within the catalyst, independent of the silica particles. The addition of iron nitrate had a large effect on the catalysts' surface area, which increased by over $100 \mathrm{~m}^{2} \mathrm{~g}^{-1}$, but did not correlate well with silica surface area. 


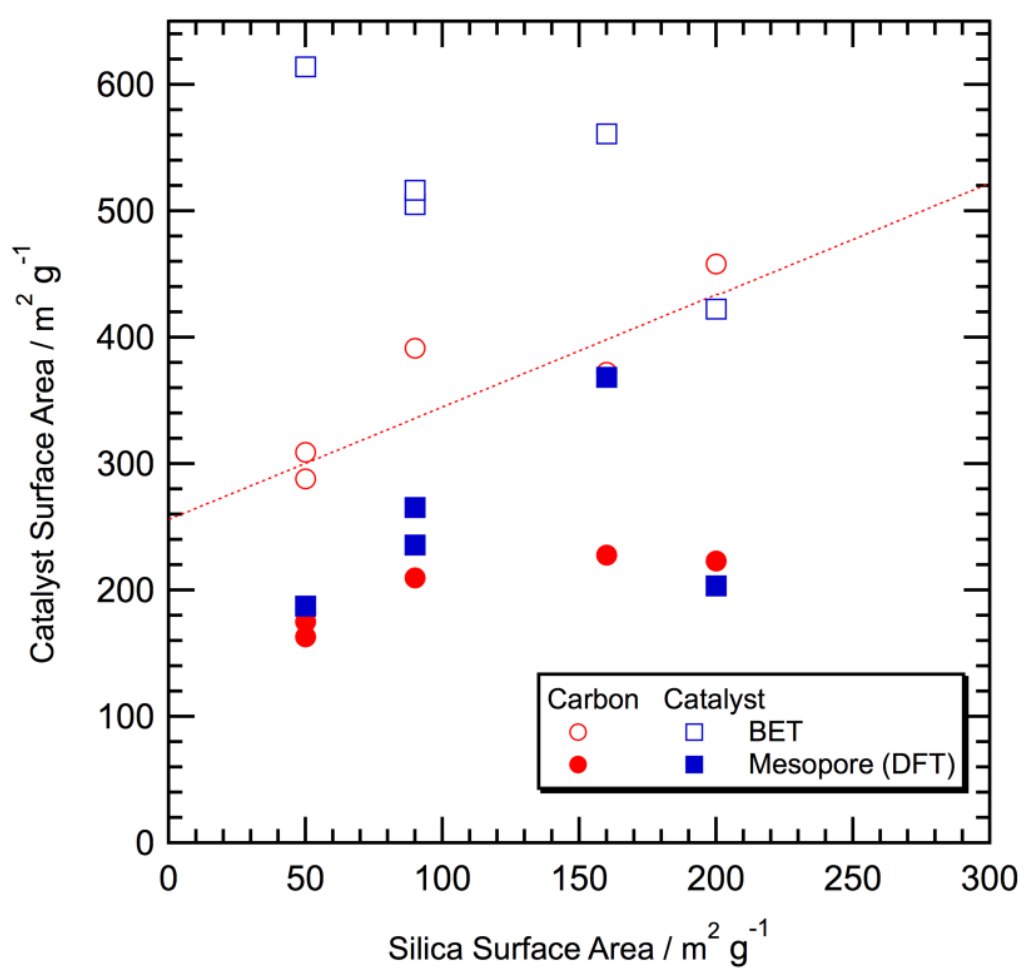

Figure 5. Surface area comparison of hard-template materials with their silica precursors. The regression line is for the porous carbon. The dotted regression line corresponds to the relation

SEM imaging provided qualitative support for the BJH and DFT pore size distributions, as shown in Figures 6 and 7 for the carbons and catalysts, respectively. The SEM images showed dark, semi-circular pore regions with a distribution of diameters. The pore diameters visually decreased with decreasing silica particle diameter, as expected from the pore size distributions. As shown in Figure 7, the catalysts generally had morphologies similar to the carbon materials, but with more structured surfaces, likely due to increased carbonization resulting from the presence of iron species. 

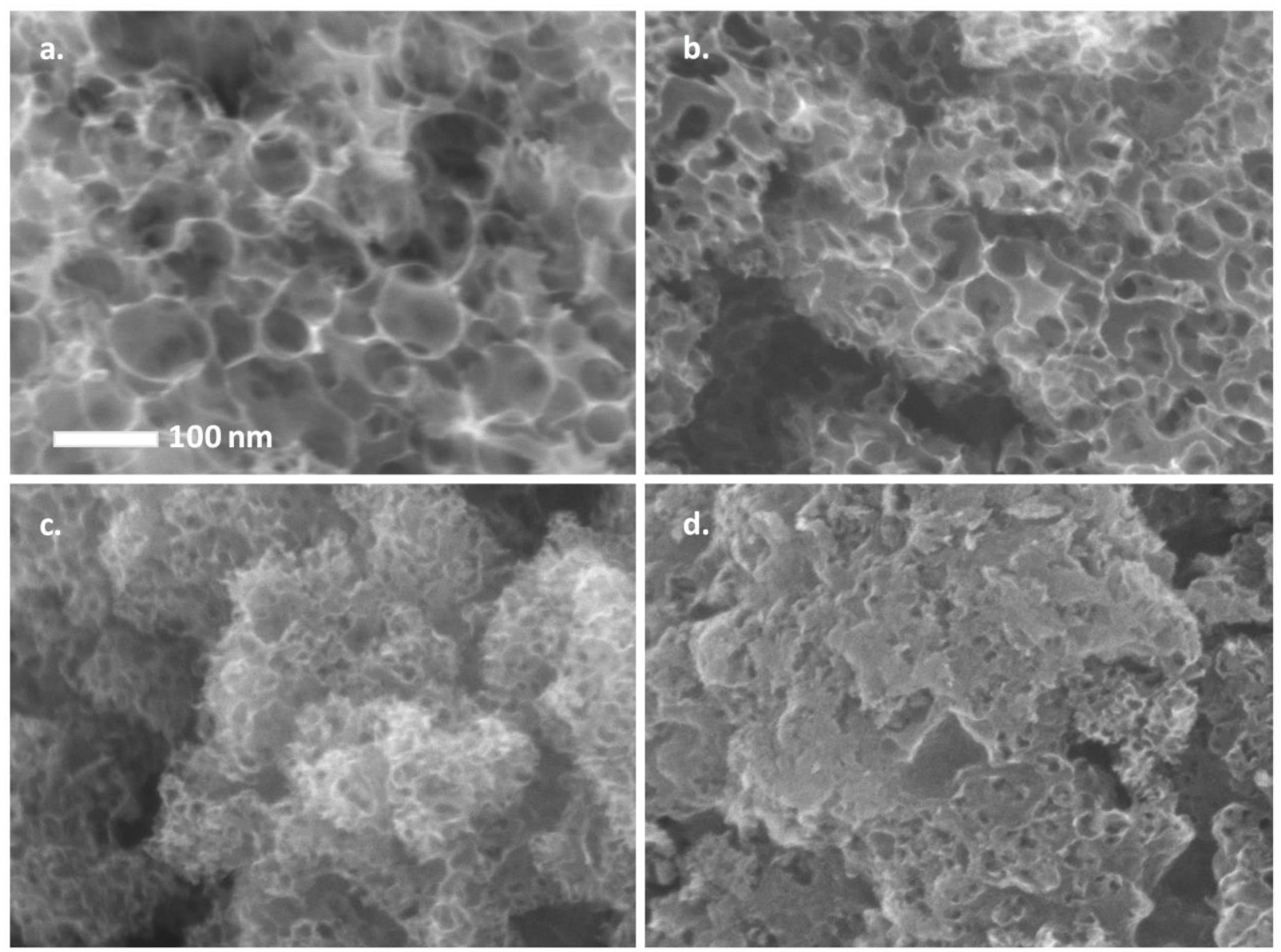

Figure 6. SEM images of the porous carbons from pyrolysis of nicarbazin on a silica template. The precursors are (a) Aerosil OX-50, (b) Cab-O-Sil L-90, (c) Cab-O-Sil LM-150, and (d) Cab-O-Sil M5P. All images have the same scale. 

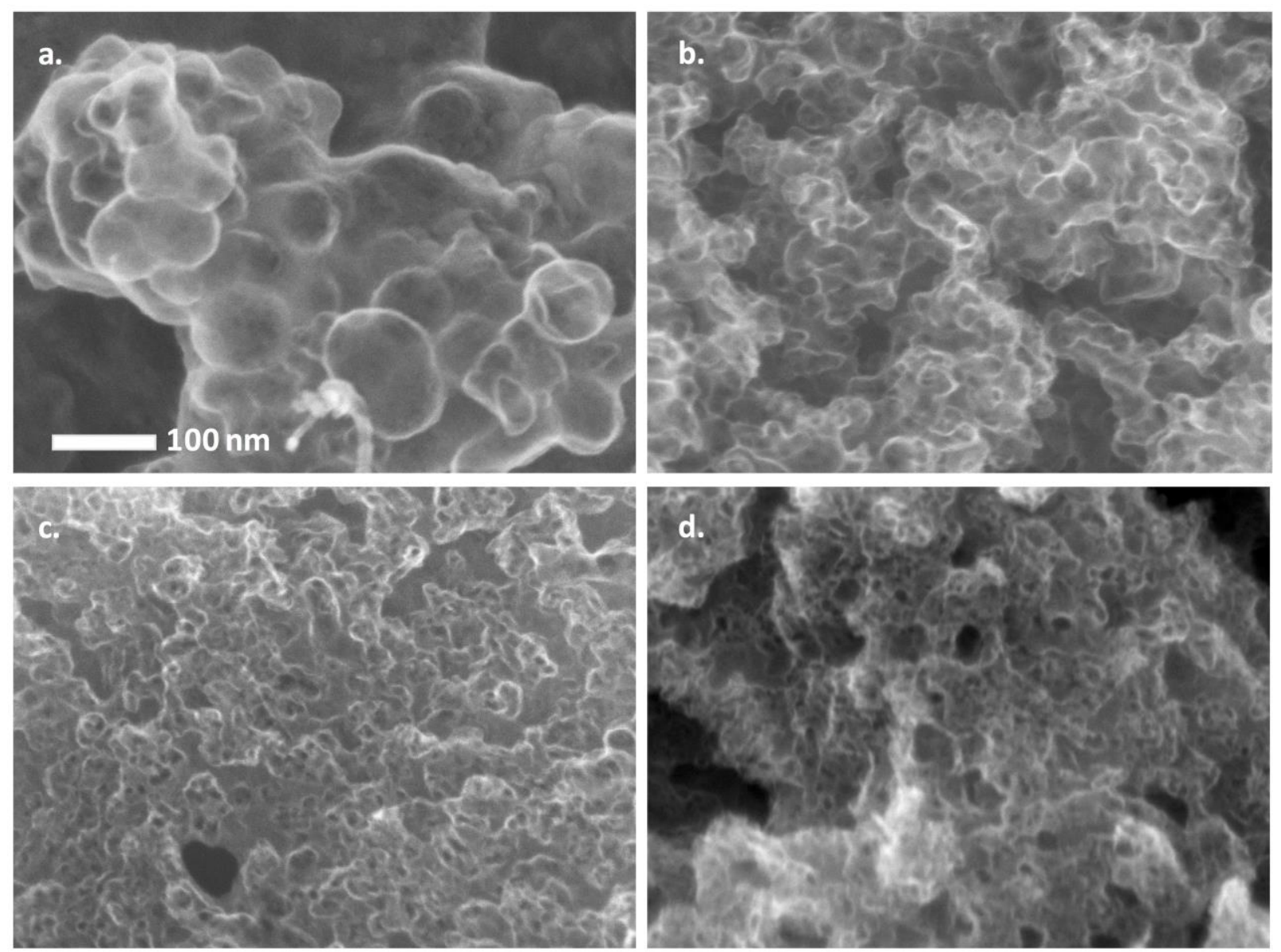

Figure 7. SEM images of the catalysts from pyrolysis of nicarbazin and iron acetate on a silica template. The precursors are (a) Aerosil OX-50, (b) Cab-O-Sil L-90, (c) Cab-O-Sil LM-150, and (d) CabO-Sil M5P. All images have the same scale.

MEA data for the catalysts are presented in Figure 8. The porous carbon materials showed minimal activity compared to the catalysts. Data for the M5P carbon material is included in Figure 8 as a representative sample for comparison. All four catalysts showed similar kinetic properties with open circuit potentials near 0.9 Volts. The major difference was observed in the low potential, transport limited regime. In this region, the LM150-templated catalyst showed optimum performance, with a catalyst pore diameter near $9 \mathrm{~nm}$ and a silica particle diameter around $18 \mathrm{~nm}$. 


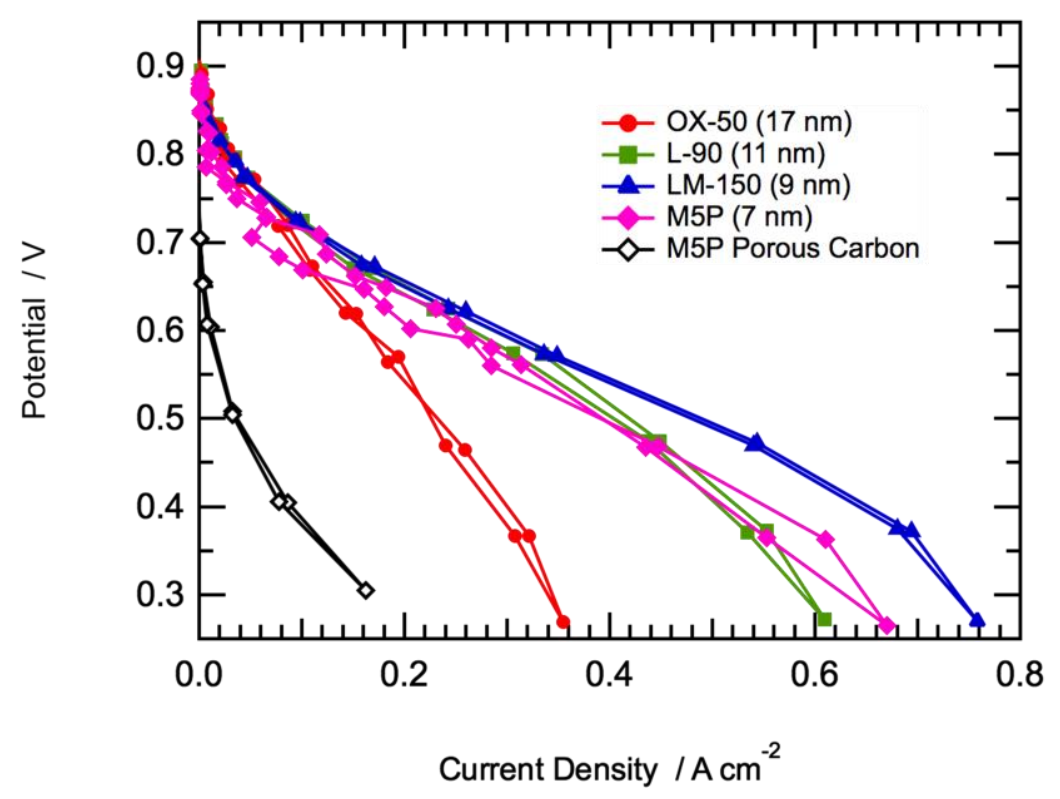

Figure 8. Membrane electrode assembly (MEA) results for the catalysts in air. The tests were performed at $80^{\circ} \mathrm{C}$, with the following conditions: 211 Nafion, $45 \mathrm{wt} \%, 1100 \mathrm{EW}, 4 \mathrm{mg} / \mathrm{cm}^{2}$, $25 \mathrm{BC}$ GDL, $100 \% \mathrm{RH}, 30 \mathrm{psig}$. The current density is non-IR corrected. The length scales represent the predominant catalyst pore size. The M5P data is representative of the other porous carbon materials.

Figure 9 demonstrates the optimum catalyst pore diameter at $0.65 \mathrm{~V}$, a potential near peak power density which is of interest for automotive applications. This optimum suggests that tuning pore size directly impacts electrode performance. Figure 9 also shows a maximum mesopore ( $2 \mathrm{~nm}-50 \mathrm{mn}$ ) surface area corresponding to the same 9-nm pore diameter as the optimum MEA performance. This correlation suggests that mesopores have a strong influence on catalyst performance, possibly due to their control over the number of accessible active sites and reactant transport to the active sites. 


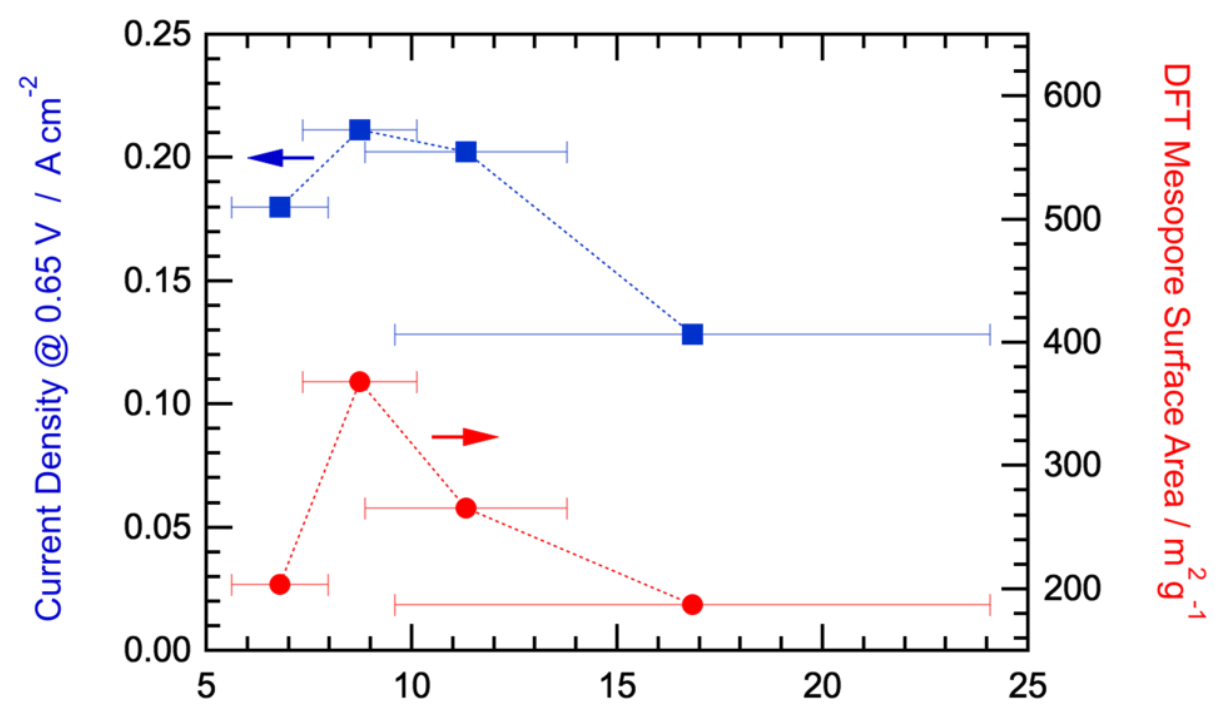

Catalyst BJH Pore Diameter / nm

Figure 9. Effect of pore size on catalyst MEA performance and mesopore surface area. The MEA current density is taken from figure 7 at $0.65 \mathrm{~V}$, with MEA tests performed at $80^{\circ} \mathrm{C}$, with the following conditions: 211 Nafion, $45 \mathrm{wt} \%, 1100 \mathrm{EW}, 4 \mathrm{mg} / \mathrm{cm}^{2}, 25 \mathrm{BC}$ GDL, $100 \% \mathrm{RH}, 30$ psi. Error bars reflect peak widths from Fig. 3.

\section{Modeling}

Several models were developed to gain insight into potential pore formation mechanisms. The models describe catalyst pore shrinkage as the collapse of unstable, graphitic pores. Previous hard template catalysts have shown a large graphitic content [9]. We speculate that formation of graphitic regions on the surface of spherical silica particles would result in significant curvature, straining the $\mathrm{sp}_{2}$ carbon bonds. Upon silica removal, bond strain would cause the pores to collapse to a more stable shape, which we chose to be a cylinder. A schematic representation of the pore collapse is presented in Figure 10. 

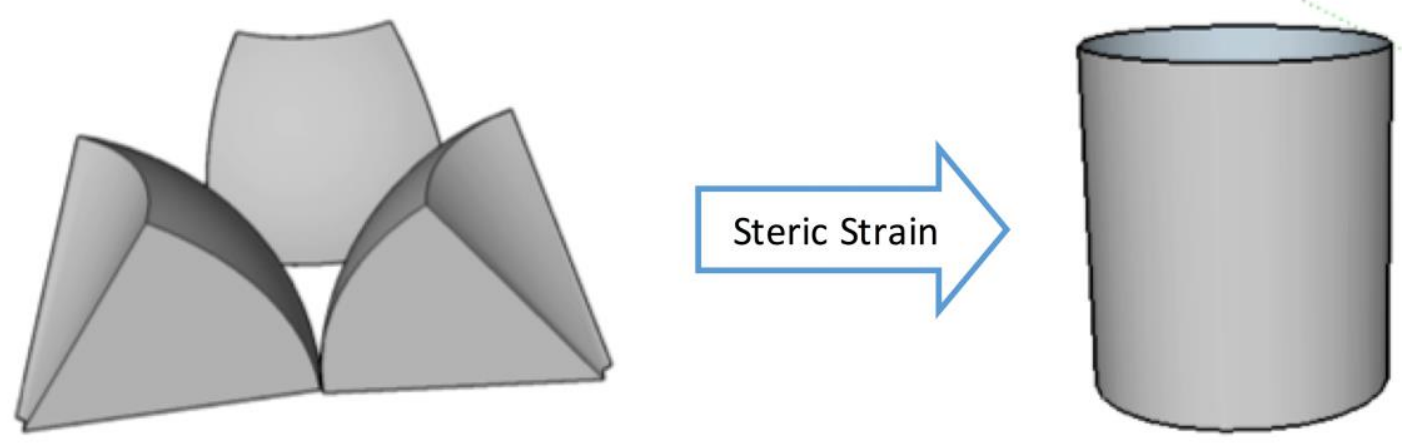

Figure 10. Schematic representation of hard template pore collapse.

Two types of models were constructed for cylindrical pore formation, the interior and exterior models, respectively, with details given in Appendix A (Supporting Information). The interior pore model assumes that the catalyst's pores form in the vacated silica spaces, whereas the exterior pore model assumed that interparticle spaces form the catalyst's pores. The exterior pore model had two variations based on cubic and hexagonal silica packing, respectively. All three models assume that both pore length and surface area are conserved upon collapse to a cylinder, and that silica particles act as uniform, hard body spheres with a regular packing scheme. The carbon-nitrogen precursor and the catalyst were assumed to form a thin layer on the silica particle's surface with a negligible thickness. Whereas carbonization is known to generate a substantial volume of micropores, the present models only consider mesopore formation. Finally, pore collapse and rearrangement was assumed to occur without mass loss. Figure 11 summarizes the models and compares them with the experimental data. 


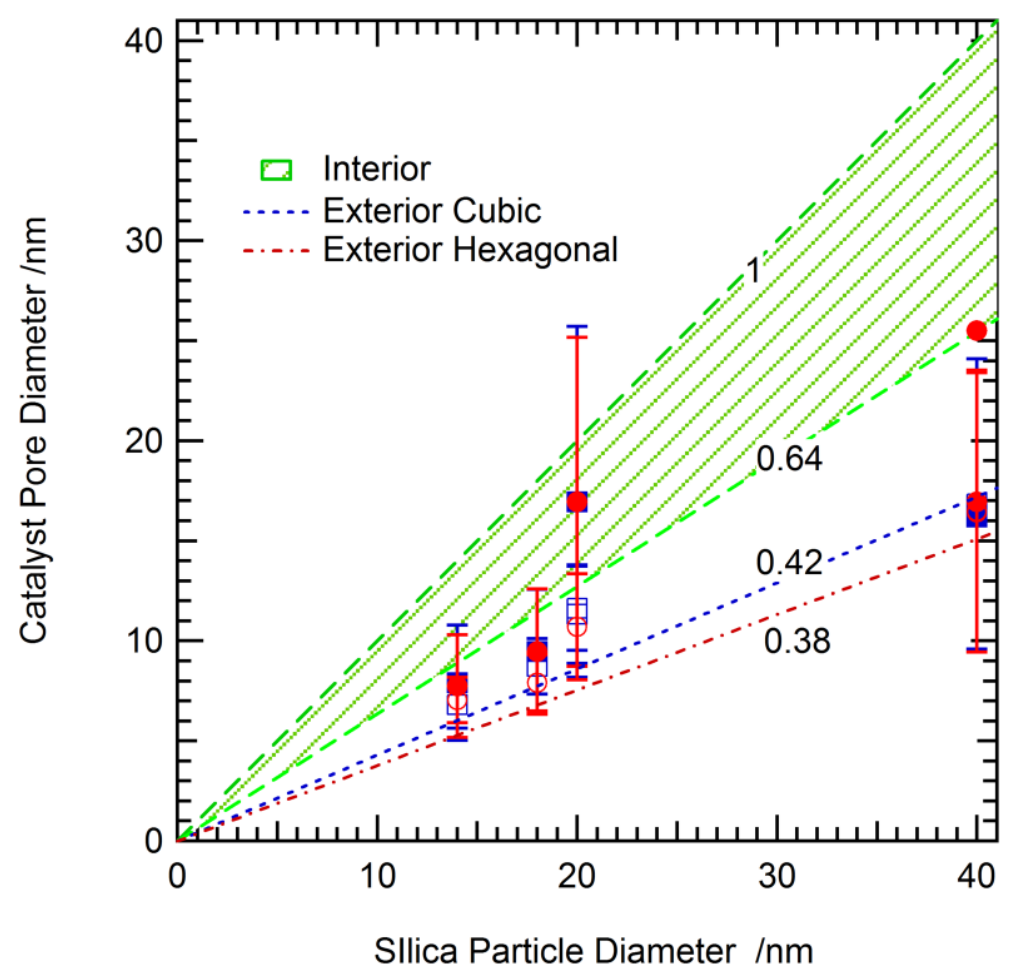

Figure 11. Comparison of the three pore formation models with experimental data. The green shaded region represents the range of values possible for the interior pore model.

None of the models provided a satisfactory fit to the experimental data. The exterior cubic packing model showed the best fit, but was still well below the experimental data. Despite the failure of the individual models to reflect the experimental data, it is noteworthy that all data points fell between the interior and exterior models. Such a fact supports the logical notion of multiple pore formation mechanisms. In the highly heterogeneous environment of pore formation, the catalyst would likely form both interior and exterior pores. Each type of pore would produce its own pore distribution, which, when added together, would create a cumulative distribution with pores sizes between the exterior and interior pore sizes. Multiple pore formation mechanisms would also provide an explanation for multiple peaks in the DFT distributions (Fig. 2b), which would result from the summation of pore distributions. Multiple formation 
mechanisms could also explain the L90 BJH-DFT discrepancy. As discussed previously, the BJH-DFT discrepancy likely results from small, bottle neck pores restricting the desorption of larger pores. Such a scenario fits well with the idea of smaller exterior pores restricting access to larger interior pores.

\section{Conclusions}

Catalysts and porous carbons materials synthesized from nicarbazin using the hardtemplate process showed high mesoporosity and exceptional oxygen reduction activity. The pore size of the hard template catalysts and porous carbons correlated well with precursor particle size, with most catalysts and porous carbon materials displaying pore diameters approximately half their precursor particle diameters. The pore size decrease suggests pore collapse during either the silica etching process or the second heat treatment. Models were developed for three pore collapse mechanisms. The experimentally-determined pore diameters fell between the interior and exterior pore models, suggesting multiple pore formation mechanisms.

\section{Acknowledgement}

We gratefully acknowledge financial support from the U.S. Department of Energy (EERE), under a Non PGM Catalyst development effort (Contract no EE 0000459) lead by Northeastern University (Prof. Sanjeev Mukerjee, P.I). 


\section{References}

[1] F. Jaouen, J. Herranz, M. Lefèvre, J.-P. Dodelet, U.I. Kramm, I. Herrmann, P. Bogdanoff, J. Maruyama, T. Nagaoka, A. Garsuch, J.R. Dahn, T. Olson, S. Pylypenko, P. Atanassov, E. a Ustinov, ACS Appl. Mater. Interfaces 1 (2009) 1623-39.

[2] F. Jaouen, E. Proietti, M. Lefèvre, R. Chenitz, J.-P. Dodelet, G. Wu, H.T. Chung, C.M. Johnston, P. Zelenay, Energy Environ. Sci. 4 (2011) 114.

[3] D.C. Higgins, Z. Chen, Can. J. Chem. Eng. 91 (2013) 1881-1895.

[4] E. Proietti, F. Jaouen, M. Lefèvre, N. Larouche, J. Tian, J. Herranz, J.-P. Dodelet, Nat. Commun. 2 (2011) 416.

[5] H.A. Gasteiger, N.M. Marković, Science (80-. ). 324 (2009) 48-49.

[6] J.H. Knox, B. Kaur, G.R. Millward, J. Chromatogr. A 352 (1986) 3-25.

[7] J. Lee, J. Kim, T. Hyeon, Adv. Mater. 18 (2006) 2073-2094.

[8] Y. Xia, Z. Yang, R. Mokaya, in:, D. Bruce, D. O’Hare, R. Walton (Eds.), Porous Mater., John Wiley \& Sons, Ltd, Chichester, UK, 2010, pp. 217-264.

[9] S. Pylypenko, S. Mukherjee, T.S. Olson, P. Atanassov, Electrochim. Acta 53 (2008) 7875-7883.

[10] A. Serov, M.H. Robson, K. Artyushkova, P. Atanassov, Appl. Catal. B Environ. 127 (2012) 300-306.

[11] M.H. Robson, A. Serov, K. Artyushkova, P. Atanassov, Electrochim. Acta 90 (2013) 656665.

[12] A. Serov, K. Artyushkova, P. Atanassov, Adv. Energy Mater. 4 (2014).

[13] S. Stariha, K. Artyushkova, A. Serov, P. Atanassov, Int. J. Hydrogen Energy (2015).

[14] A. Serov, K. Artyushkova, E. Niangar, C. Wang, N. Dale, F. Jaouen, M.-T. Sougrati, Q. Jia, S. Mukerjee, P. Atanassov, Nano Energy 16 (2015) 293-300.

[15] D. Boldridge, Aerosol Sci. Technol. 44 (2010) 182-186.

[16] S. Brunauer, P.H. Emmett, E. Teller, J. Am. Chem. Soc. 60 (1938) 309-319.

[17] E.P. Barrett, L.G. Joyner, P.P. Halenda, J. Am. Chem. Soc. 73 (1951) 373-380.

[18] E.A. Ustinov, D.D. Do, V.B. Fenelonov, Carbon N. Y. 44 (2006) 653-663.

[19] E.A. Ustinov, Langmuir 24 (2008) 6668-6675.

[20] K. Sing, D. Everet, R. Haul, L. Moscou, R. Pierotti, J. Rouquerol, T. Siemieniewska, Pure Appl. Chem. 57 (1985) 603-619.

[21] O.W. Flörke, H.A. Graetsch, F. Brunk, L. Benda, S. Paschen, H.E. Bergna, W.O. Roberts, W.A. Welsh, C. Libanati, M. Ettlinger, D. Kerner, M. Maier, W. Meon, R. Schmoll, H. Gies, D. Schiffmann, in:, Ullmann's Encycl. Ind. Chem., Wiley-VCH Verlag GmbH \& Co. KGaA, 2000.

[22] Y. Yang, K. Chiang, N. Burke, Catal. Today 178 (2011) 197-205.

[23] J.H. de Boer, A. Van Den Heuvel, B.G. Linsen, J. Catal. 3 (1964) 268-273. 
[24] D.B. Braun, M.R. Rosen, Rheology Modifiers Handbook - Practical Use and Application, William Andrew Publishing, 2000.

[25] Y. Li, M. Hariharaputhiran, S. V. Babu, J. Mater. Res. 16 (2001) 1066-1073. 

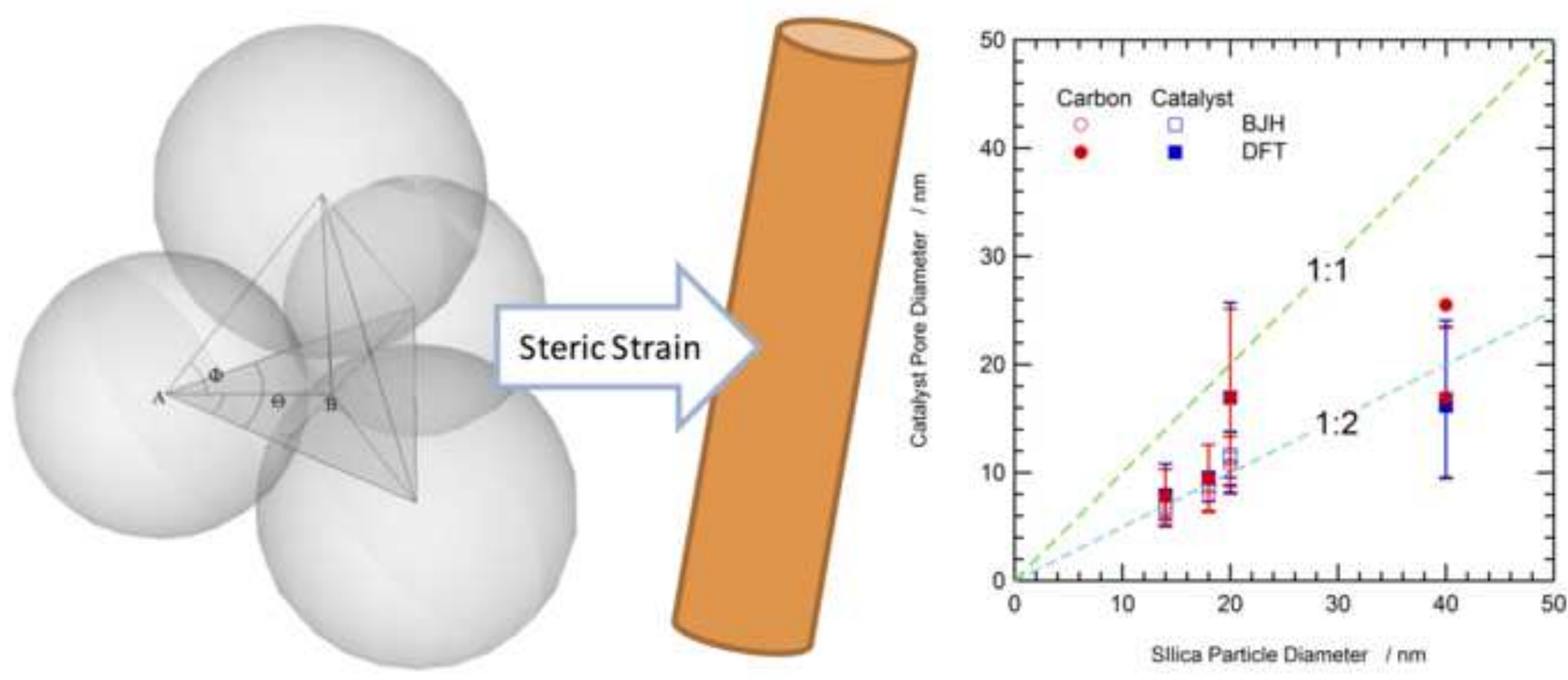